\title{
Atypical Location and Morphology of a Proximal Nail Fold Nevus Mimicking Other Diseases
}

\author{
Renan Minotto Andréia Della Giustina Leandro Damiani Ana Letícia Boff \\ Karen Scherer Bastos Bianca Coelho Furtado \\ Clinical and Surgical Nail Disease Outpatient Clinic, Division of Dermatology, Santa Casa de Misericórdia, \\ Porto Alegre, Brazil
}

\section{Key Words}

Complex surgery of the nails - Melanocytic nevus .

Melanoma $\cdot$ Nail $\cdot$ Intradermal nevus $\cdot$ Nail tumors

\section{Abstract}

Melanocytic nevi result from the proliferation of melanocytes at the dermal-epidermal junction and/or in the dermis and very rarely affect the nail matrix and bed. Intradermal nevi are tumors often diagnosed in routine clinical dermatological practice, with typical clinical and histopathologic aspects, and found in a wide variety of skin sites. We report a case of intradermal melanocytic nevus of the proximal nail fold with uncommon intraoperative gross findings and without involvement of the nail bed or matrix, showing intradermal component only, which draws our attention to the possible different presentation of melanocytic lesions. No description of intradermal nevi at this site has been reported in the literature so far.

(c) 2016 S. Karger AG, Base

\section{Introduction}

Nevi are characterized by the proliferation of melanocytes shaped like nevus cells and grouped into nests. These nests can be found in the epidermis or in the epithelium of the nail bed and matrix (junctional nevi), in the dermis (intradermal nevi), or at both sites (compound nevi) [1]. They develop from childhood to adulthood and may be found in different parts of the body; however, some sites are unusual, as indicated by low incidence rates, as is the case of the nail matrix and nail bed [2]. We describe the case of a patient with a benign lesion frequently observed in clinical practice, but with an uncommon intraoperative gross finding on the proximal nail fold of the left second digit, mimicking other diseases such as nail matrix melanocytic nevus and nail apparatus melanoma.

\section{Case Report}

A female 56-year-old patient presented with an asymptomatic lesion on her left second digit that had been growing slowly for 3 years (fig. 1). She had no previous history of trauma or similar lesion. Dermatoscopic examination (fig. 2) revealed a bluish macu-

\section{KARGER}

E-Mail karger@karger.com www.karger.com/sad
C 2016 S. Karger AG, Basel

2296-9195/16/0022-0045\$39.50/0
Dr. Renan Minotto

Ambulatório de Doenças das Unhas, Serviço de Dermatologia, Santa Casa de Misericórdia Rua Professor Annes Dias, 295 Centro Histórico

Porto Alegre, RS 90020-090 (Brazil)

E-Mail rminotto@gmail.com 


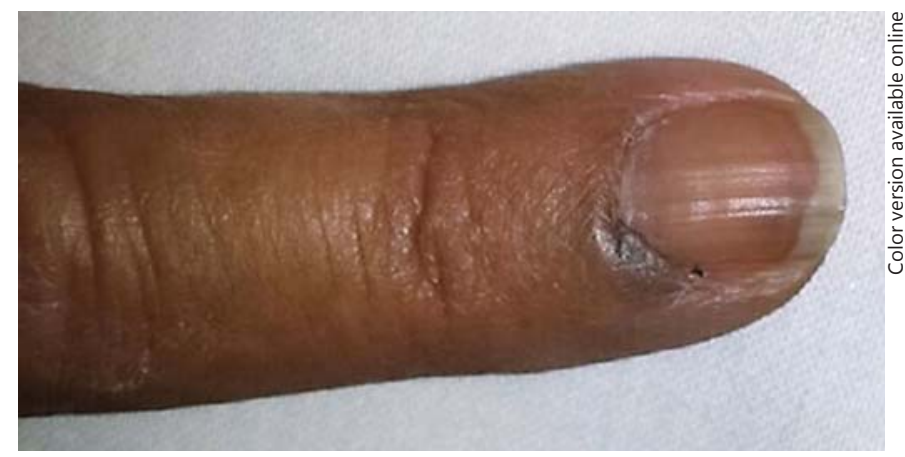

Fig. 1. Gross appearance of the lesion.

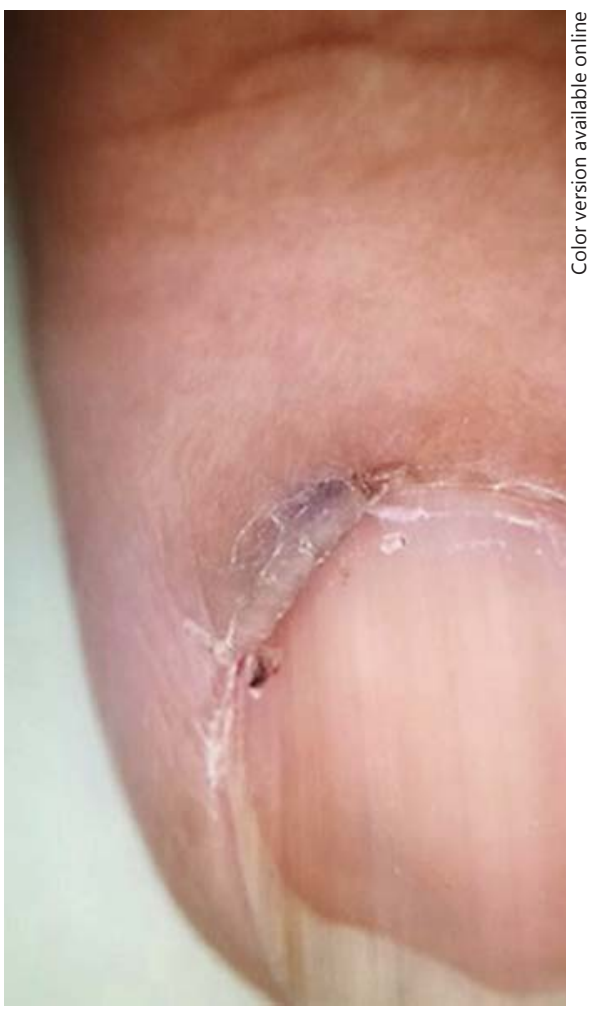

Fig. 2. Presence of irregular whitish blue areas.

lar lesion apparently confined to a small area adherent to the cuticle, suggesting pseudo-Hutchinson's sign. As malignancy with possible nail matrix involvement was clinically suspected, the lesion was surgically explored, with retraction of the nail fold for better visualization (fig. 3). The intraoperative dermatoscopic examination (fig. 4) revealed an extensive tumor-like lesion with irregular whitish blue pigmentation in most of the ventral surface of the proximal nail fold, without melanotic pigmentation in the nail matrix and in the nail plate. The histopathologic analysis showed proliferation of nests of small nevus-like cells, with pigmentation in their cytoplasm throughout the lesion. The nests were confined to the dermis and were histologically mature as the lesion extend-

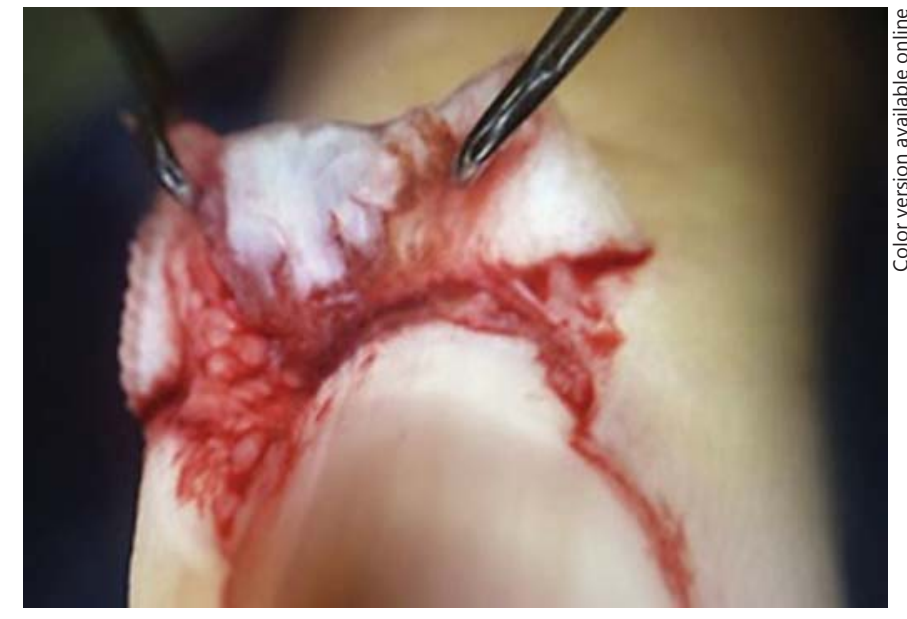

Fig. 3. Retraction of the proximal nail fold with visualization of the tumor.

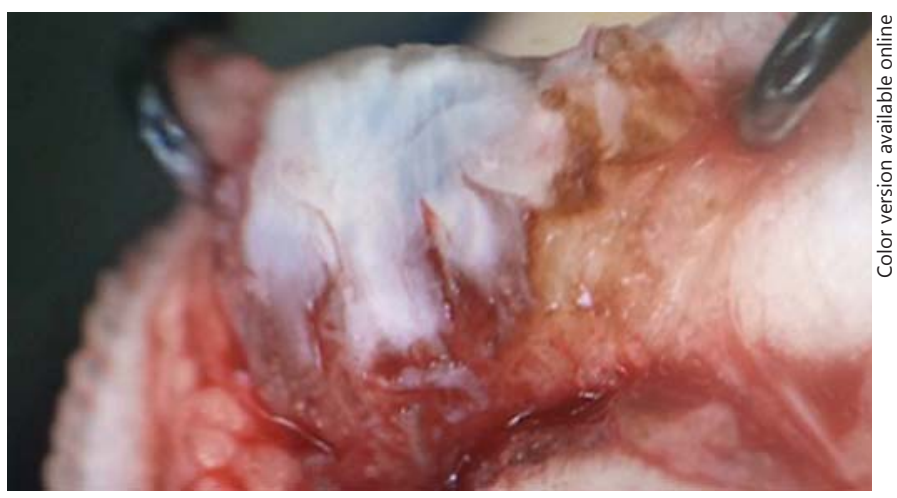

Fig. 4. Dermatoscopic examination of the lesion showing a tumorlike aspect with bluish streaks.

ed deeper into the dermis. No mitoses or atypical cytological findings were observed. The histological diagnosis indicated intradermal melanocytic nevus (fig. 5). The surgical wound edges were carefully coaptated, properly preserving the anatomical characteristics of the region (fig. 6). The postoperative period was uneventful (fig. 7).

\section{Discussion}

An intradermal nevus is, in general, raised, domeshaped, and nonpigmented [3]. It develops most commonly on the face, and there have been no reports in the literature so far about acquired intradermal nevi located on the proximal nail fold. Melanocytic nevi of the nail apparatus can be congenital or acquired and commonly arise from the nail matrix. They account for $12 \%$ of lon- 


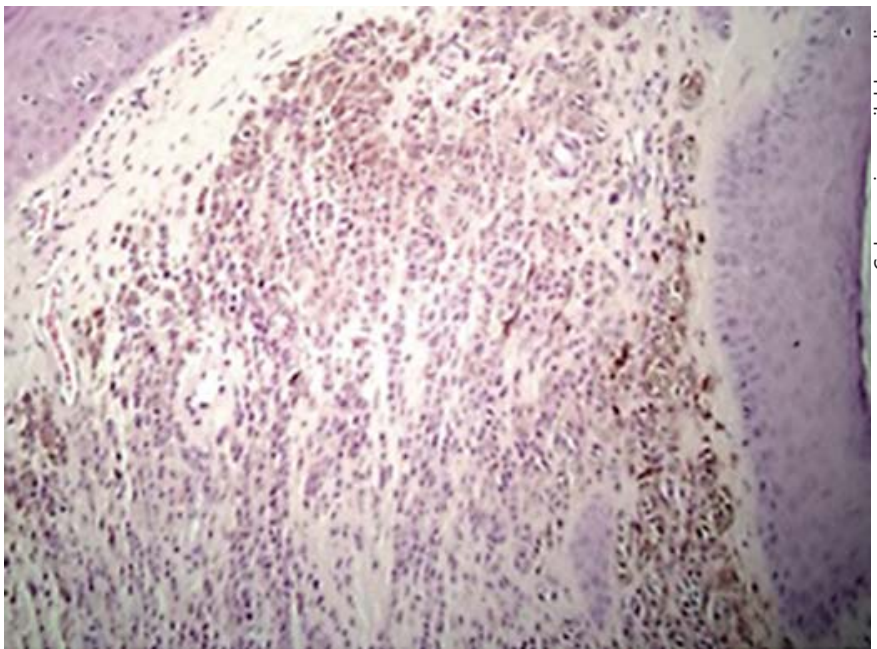

Fig. 5. Histopathologic analysis.

gitudinal melanonychia in adults and for almost $50 \%$ in children. They have a predilection for the thumb, and their size averages more than $3 \mathrm{~mm}$ in $50 \%$ of the cases. Their brownish-black color is present in two thirds of the cases, and periungual pigmentation (pseudoHutchinson's sign) is observed in one third of the cases [4]. Pseudo-Hutchinson's sign is the apparent pigmentation of the proximal nail fold seen through the transparent cuticle [2] and can be subdivided into three categories: benign, malignant nonmelanoma, and illusory. Associated benign conditions include racial melanonychia, Peutz-Jeghers syndrome, Laugier-Hunziker syndrome, drug-induced (minocycline) conditions, malnutrition, HIV, and congenital nevus. Malignant nonmelanoma conditions, such as Bowen's disease, can also manifest in the same fashion. Finally, illusory pigmentation of the cuticle occurs when pigmentation is seen in the nail matrix or bed [4]. It is essential that subungual melanoma be ruled out, as both benign and malignant lesions can manifest as a linear pigmented band along the nail plate [4]. Clinical findings suggestive of malignant lesions include irregular pigmentation, color variegation, and bands that have a late development [2]. In addition, owing to rapid growth, the bands are larger on the proximal than on the distal extremity, having a funnel shape [2]. Hutchinson's sign is not pathognomonic, but it is suggestive of melanoma and is characterized by extension of longitudinal melanonychia into the periungual tissues [3]. For better diagnostic accuracy, a dermatoscopic examination must be often conducted in the intraoperative period, with retraction of the nail

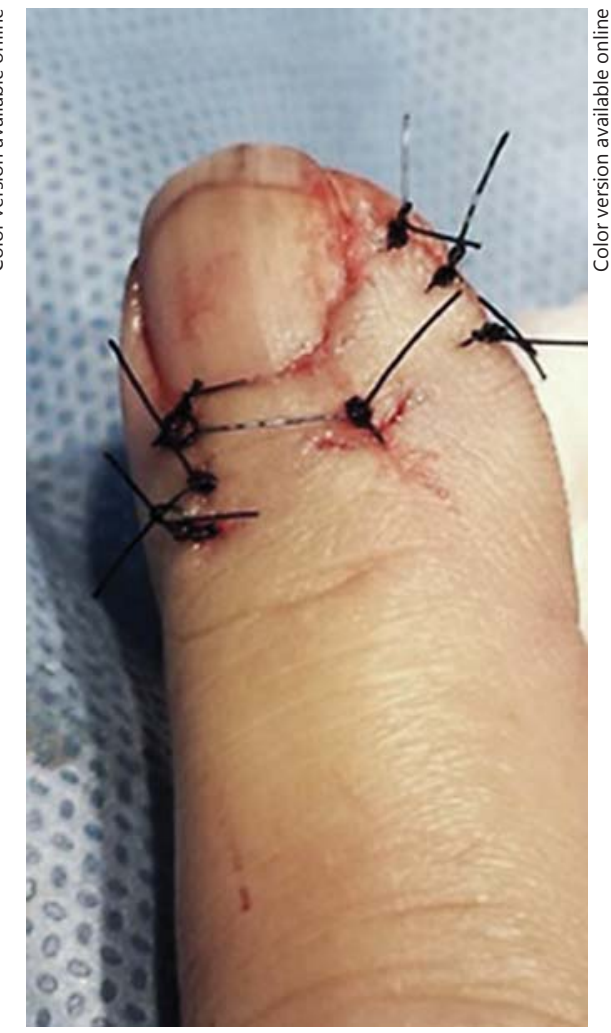

Fig. 6. Immediate postoperative period showing coaptated wound edges and preservation of the local anatomy.

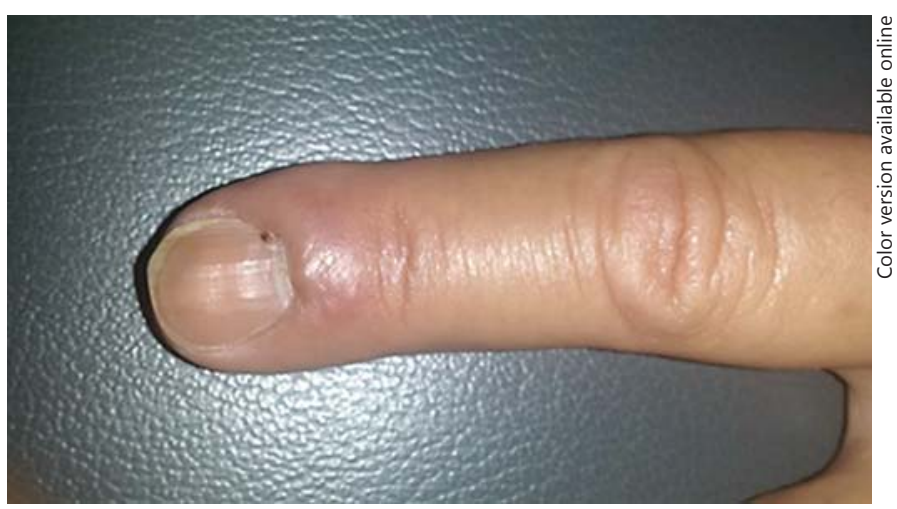

Fig. 7. Aspect in the postoperative period.

plate in case of matrix involvement. Notwithstanding, the histopathologic analysis is crucial to confirm the diagnosis. Based on the clinical and intraoperative findings, other differential diagnoses had to be considered, namely blue nevus, vascular lesion, foreign body, pseudo-Hutchinson's sign, melanoma, glomus tumor, neurofibroma, fibrosis, pyogenic granuloma, myxoid cyst, 
angiofibroma, and myxoid fibroma $[2,3,5,6]$. Surgical excision of the lesion, for investigation and exclusion of malignancies, was the most appropriate therapeutic option in this case [2]. In this report, we described a new clinical presentation of intradermal nevus, showing the importance of correctly identifying nail changes that are suggestive of melanoma.

\section{Statement of Ethics}

The patient's written consent has been obtained.

\section{Disclosure Statement}

The authors certify that they have no affiliations with or involvement in any organization or entity with any financial interest.

\section{References}

1 SR Granter, McKee PH, Calonje E, et al: Melanoma associated with blue nevus and melanoma mimicking cellular blue nevus: a clinicopathologic study of 10 cases on the spectrum of so-called 'malignant blue nevus'. Am J Surg Pathol 2001;25:316-323.

2 Goldminz AM, et al: Congenital subungual melanocytic nevus with a pseudo-Hutchinson sign. Dermatol Online J 2013;19:8.

3 Bishop JAN: Lentigos, melanocytic naevi and melanoma; in Burns T, Breathnach S,
Cox N, Griffiths C (eds): Rook's Textbook of Dermatology, ed 8. Wiley-Blackwell, 2010, p 4432.

4 Josette A, Lateur N: Pigmented nail disorders. Dermatologic Clin 2006;24:329-339.

5 Lin YC, Wu YH, et al: Nail changes and association of osteoarthritis in digital myxoid cyst. Dermatol Surg 2008;34:364-369.

6 Causeret AS, Skowron F, Viallard AM, et al: Subungual blue nevus. J Am Acad Dermatol 2003;49:310-312. 